\title{
Case Report \\ Streptococcus mutans infective endocarditis complicated by vertebral discitis following dental treatment without antibiotic prophylaxis
}

\author{
Correspondence \\ Daniel P. Webster \\ danwebster@doctors.org.uk
}

Received 26 March 2010

Accepted 4 July 2010

\author{
Sujata Biswas, ${ }^{1}$ lan C. J. W. Bowler, ${ }^{2}$ Christopher Bunch, ${ }^{1}$ \\ Bernard Prendergast ${ }^{3}$ and Daniel P. Webster ${ }^{2}$ \\ ${ }^{1}$ Department of Acute General Medicine, John Radcliffe Hospital, Oxford OX3 9DU, UK \\ ${ }^{2}$ Department of Microbiology, John Radcliffe Hospital, Oxford OX3 9DU, UK \\ ${ }^{3}$ Department of Cardiology, John Radcliffe Hospital, Oxford OX3 9DU, UK
}

\begin{abstract}
We report what we believe is the first reported case of Streptococcus mutans endocarditis complicated by vertebral discitis. The case is particularly interesting and topical as it occurred in a patient with pre-existing cardiac valvular disease who had recently had a dental procedure without antibiotic prophylaxis following a dramatic shift in the UK guidelines.
\end{abstract}

\section{Case report}

A 67-year-old man presented with a 3 week history of general malaise, night sweats, anorexia and $6 \mathrm{~kg}$ weight loss. Over the preceding 2 months, he had developed lower back pain of increasing severity, without lower limb weakness, paraesthesia or incontinence. His past medical history included mitral valve prolapse with associated moderate regurgitation diagnosed 3 years previously and several dental procedures, the most recent of which entailed drainage of a dental abscess 3 months prior to admission. Following the diagnosis of mitral valve disease, antibiotic prophylaxis with oral clindamycin (as the patient had previously developed a rash with oral amoxicillin) had been administered before all dental procedures. However, following a recent change in the infective endocarditis (IE) prevention guidelines in the UK, this had been omitted for the most recent procedure. There was also a history of hypertension and benign prostatic hypertrophy and the patient's regular medications included ramipril, simvastatin and solifenacin. On examination, the patient was afebrile, there was a pansystolic murmur radiating to the axilla and no peripheral stigmata of IE. There was lumbar spinal tenderness and neurological examination of the legs was normal. Blood tests revealed a haemoglobin of $11.8 \mathrm{~g}$ $\mathrm{dl}^{-1}$ and white cell count of $16.24 \times 10^{9} \mathrm{l}^{-1}$ (neutrophils $75 \%$ ). C-reactive protein was $99 \mathrm{mg} \mathrm{l}^{-1}$. Plain X-ray of the lumbar spine showed degenerative changes only but T2weighted magnetic resonance imaging showed increased signal at the L5/S1 disc space and adjacent endplates, highly suggestive of discitis (see Fig. 1). Transthoracic echocardiogram showed a thickened mitral valve with bileaflet

Abbreviation: IE, infective endocarditis. prolapse, moderate mitral regurgitation and a mass attached to the posterior leaflet. Subsequent transoesophageal imaging confirmed these findings, the mass being highly suggestive of a large vegetation. The diagnosis of IE complicated by vertebral discitis was made.

Five sets of blood cultures were taken over 3 days and became positive within $48 \mathrm{~h}$ of incubation. The initial Gram stain showed a Gram-positive staining rod (see Fig. 2), occasionally forming chains. The cultures grew Streptococcus mutans identified by the API 20 Strep identification kit (bioMérieux) in our laboratory and confirmed by the Health Protection Agency UK Streptococcal Reference Laboratory. MICs for penicillin $\left(0.032 \mathrm{mg} \mathrm{l}^{-1}\right)$ and ceftriaxone $\left(0.094 \mathrm{mg} \mathrm{l}^{-1}\right)$ were determined by E-test $(\mathrm{AB}$ Biodisk).

Taking into acount the history of allergy to penicillin, the patient was treated with intravenous ceftriaxone $(2 \mathrm{~g}$ once daily for 6 weeks) and gentamicin (80 mg twice daily for the first 2 weeks).

After 2 weeks of therapy, the white cell count had normalized and the C-reactive protein had fallen from 99 to $15 \mathrm{mg} \mathrm{l}^{-1}$. There were no cardiac symptoms, no haemodynamic compromise and serial echocardiography demonstrated reduced size of the vegetation with no increase in the severity of mitral regurgitation. His lumbar back pain had improved and the patient was able to mobilize comfortably. He was discharged to complete the remaining 4 weeks of intravenous ceftriaxone as outpatient parenteral therapy followed by 6 weeks of oral clindamycin $(300 \mathrm{mg}$ four times daily). He made a good recovery and repeat blood cultures following completion of antibiotic therapy were sterile. 


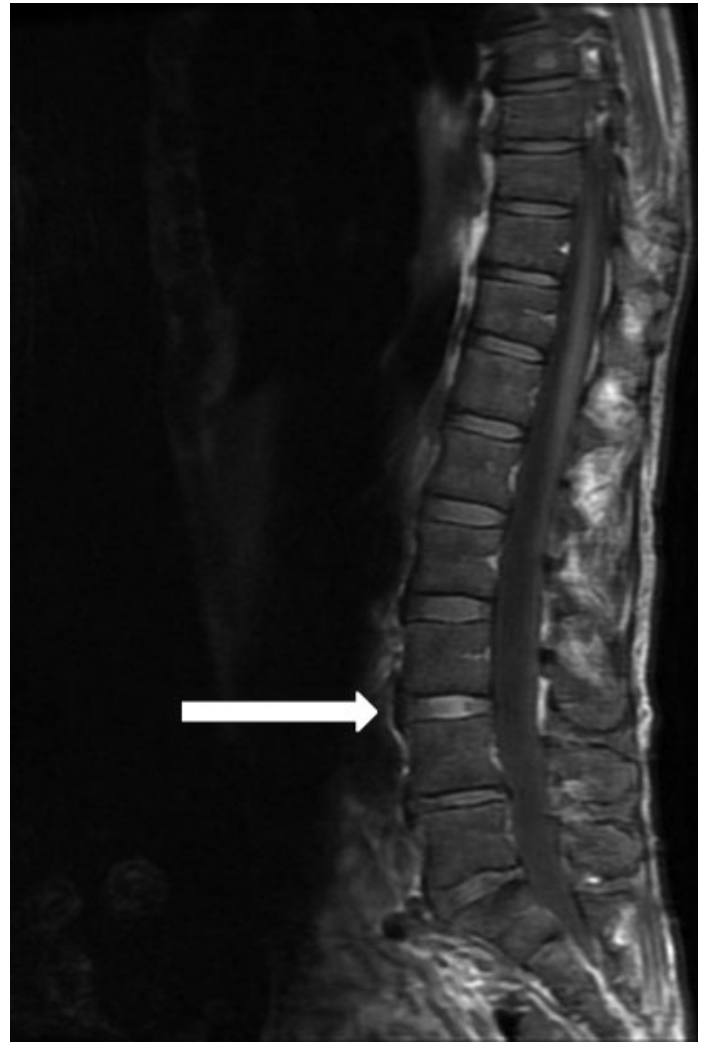

Fig. 1. T2-weighted magnetic resonance imaging of the lumbar spine showing increased signal at the L5/S1 disc space and adjacent endplates, highly suggestive of discitis.

\section{Discussion}

S. mutans is a commensal of the oral cavity and considered a primary cause of dental caries. The organism can survive in the bloodstream and has been reported as a cause of IE (Jung et al., 2009). Several virulence factors have been identified for IE including a fibronectin-binding protein

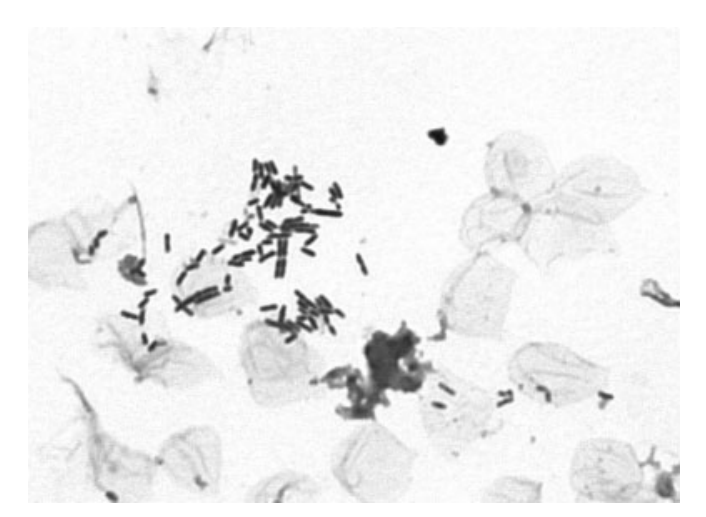

Fig. 2. Gram stain of blood cultures showing pelomorphic Gram positive-staining organisms.
(AtlA autolysin) (Jung et al., 2009), serotype-specific polysaccharides associated with phagocytosis resistance (Nakano \& Ooshima, 2009) and cell surface protein antigen c, which is involved in platelet aggregation (MatsumotoNakano et al., 2009).

Cells of S. mutans are non-haemolytic Gram-positive cocci that typically appear rod-shaped in acid media and can be mistaken as contaminating 'diphtheroids'. They show a more streptococcal appearance (cocci in chains) when subcultured on neutral media (Schelenz et al., 2005). Conventional laboratory identification techniques can misidentify them and 16S rRNA gene sequencing may be required for formal identification (Petti et al., 2005; Schelenz et al., 2005).

Dental procedures may be associated with transient bacteraemia. However, there is no clear evidence that the prior administration of antibiotic prophylaxis is either effective or ineffective in preventing IE, or whether the potential harms and costs of prophylaxis outweigh any potential benefit (Oliver et al., 2008). Additionally, there is evidence that cumulative exposure to bacteraemia is significantly greater from everyday procedures (such as tooth brushing) when compared to isolated invasive dental procedures (Roberts, 1999). Following literature reviews, the American Heart Association (AHA), UK National Institute for Health and Clinical Excellence (NICE) and European Society of Cardiology (ESC) published new guidelines for the prevention of IE (in 2007, 2008 and 2009, respectively) (Habib et al., 2009; Richey et al., 2008; Wilson et al., 2007). Although all agree that the benefits of prophylaxis are unproven, the NICE guidelines recommend no prophylaxis for dental procedures for any patients 'at risk' of IE, whereas the AHA and ESC guidelines continue to recommend prophylaxis for 'high risk' patients with the poorest outcomes from IE (Richey et al., 2008; Wilson et al., 2007).

To our knowledge, this is the first reported case of $S$. mutans IE complicated by discitis. There was a clear temporal association with a dental procedure at an infected site which, following a dramatic change in guidelines, was not accompanied by antibiotic prophylaxis. The current UK NICE guidelines do not recommend antibiotic prophylaxis for any dental procedure whereas, in contrast, for gastro-intestinal or genito-urinary tract procedures prophylaxis is recommended at a site where infection is suspected (Richey et al., 2008). In light of this case, we believe that there is an argument for recommending prophylaxis for dental procedures where infection is involved. Additionally, the question of whether our patient should receive antibiotic prophylaxis for future dental procedures remains contentious, particularly while there is discrepancy between national and international guidelines. However, following this experience, we are recommending continuing antibiotic prophylaxis for future dental procedures in our patient. 


\section{References}

Habib, G., Hoen, B., Tornos, P., Thuny, F., Prendergast, B., Vilacosta, I., Moreillon, P., de Jesus Antunes, M., Thilen, U. \& other authors (2009). Guidelines on the prevention, diagnosis, and treatment of infective endocarditis (new version 2009): the Task Force on the Prevention, Diagnosis, and Treatment of Infective Endocarditis of the European Society of Cardiology (ESC). Eur Heart J 30, 2369-2413.

Jung, C. J., Zheng, Q. H., Shieh, Y. H., Lin, C. S. \& Chia, J. S. (2009). Streptococcus mutans autolysin AtlA is a fibronectin-binding protein and contributes to bacterial survival in the bloodstream and virulence for infective endocarditis. Mol Microbiol 74, 888-902.

Matsumoto-Nakano, M., Tsuji, M., Inagaki, S., Fujita, K., Nagayama, K., Nomura, R. \& Ooshima, T. (2009). Contribution of cell surface protein antigen c of Streptococcus mutans to platelet aggregation. Oral Microbiol Immunol 24, 427-430.

Nakano, K. \& Ooshima, T. (2009). Serotype classification of Streptococcus mutans and its detection outside the oral cavity. Future Microbiol 4, 891-902.

Oliver, R., Roberts, G. J., Hooper, L. \& Worthington, H. V. (2008), Antibiotics for the prophylaxis of bacterial endocarditis in dentistry. Cochrane Database Syst Rev CD003813.
Petti, C. A., Polage, C. R. \& Schreckenberger, P. (2005). The role of $16 \mathrm{~S}$ rRNA gene sequencing in identification of microorganisms misidentified by conventional methods. J Clin Microbiol 43, 61236125.

Richey, R., Wray, D. \& Stokes, T. (2008). Prophylaxis against infective endocarditis: summary of NICE guidance. BMJ 336, 770-771.

Roberts, G. J. (1999). Dentists are innocent! "Everyday" bacteremia is the real culprit: a review and assessment of the evidence that dental surgical procedures are a principal cause of bacterial endocarditis in children. Pediatr Cardiol 20, 317-325.

Schelenz, S., Page, A. J. \& Emmerson, A. M. (2005). Streptococcus mutans endocarditis: beware of the 'diphtheroid'. J R Soc Med 98, 420-421.

Wilson, W., Taubert, K. A., Gewitz, M., Lockhart, P. B., Baddour, L. M., Levison, M., Bolger, A., Cabell, C. H., Takahashi, M. \& other authors (2007). Prevention of infective endocarditis: guidelines from the American Heart Association: a guideline from the American Heart Association Rheumatic Fever, Endocarditis, and Kawasaki Disease Committee, Council on Cardiovascular Disease in the Young, and the Council on Clinical Cardiology, Council on Cardiovascular Surgery and Anesthesia, and the Quality of Care and Outcomes Research Interdisciplinary Working Group. Circulation 116, 1736-1754. 